\title{
Renormalization scheme dependence of high-order perturbative QCD predictions
}

\author{
Yang $\mathrm{Ma}^{1, *}$ and Xing-Gang $\mathrm{Wu}^{2, \dagger}$ \\ ${ }^{1}$ Pittsburgh Particle Physics, Astrophysics, and Cosmology Center, Department of Physics and Astronomy, \\ University of Pittsburgh, 3941 O'Hara Street, Pittsburgh, Pennsylvania 15260, USA \\ ${ }^{2}$ Department of Physics, Chongqing University, Chongqing 401331, People's Republic of China
}

(Received 2 January 2018; published 27 February 2018)

\begin{abstract}
Conventionally, one adopts typical momentum flow of a physical observable as the renormalization scale for its perturbative QCD (pQCD) approximant. This simple treatment leads to renormalization schemeand-scale ambiguities due to the renormalization scheme and scale dependence of the strong coupling and the perturbative coefficients do not exactly cancel at any fixed order. It is believed that those ambiguities will be softened by including more higher-order terms. In the paper, to show how the renormalization scheme dependence changes when more loop terms have been included, we discuss the sensitivity of pQCD prediction on the scheme parameters by using the scheme-dependent $\left\{\beta_{m \geq 2}\right\}$-terms. We adopt two four-loop examples, $e^{+} e^{-} \rightarrow$ hadrons and $\tau$ decays into hadrons, for detailed analysis. Our results show that under the conventional scale setting, by including more-and-more loop terms, the scheme dependence of the pQCD prediction cannot be reduced as efficiently as that of the scale dependence. Thus a proper scale-setting approach should be important to reduce the scheme dependence. We observe that the principle of minimum sensitivity could be such a scale-setting approach, which provides a practical way to achieve optimal scheme and scale by requiring the pQCD approximate be independent to the "unphysical" theoretical conventions.
\end{abstract}

DOI: 10.1103/PhysRevD.97.036024

\section{INTRODUCTION}

Within the framework of the perturbative quantum chromodynamics (pQCD) theory, a physical observable (Q) can be expanded up to $n$th order in the strong coupling constant $\alpha_{s}$ as

$$
\varrho_{n}=\sum_{i=0}^{n} \mathcal{C}_{i}^{\mathcal{R}}(\mu)\left(a_{s}^{\mathcal{R}}\right)^{p+i}(\mu)
$$

where $\mathcal{R}$ stands for the chosen renormalization scheme, $p$ is the power of the strong coupling constant associated with the tree-level term, $a_{s}^{\mathcal{R}}(\mu)=\alpha_{s}^{\mathcal{R}}(\mu) / \pi$. Here $\mu$ is the renormalization scale, which is in principle arbitrary but should be within the perturbative region to ensure the reliability of the pQCD expansion. One usually sets the magnitude of $\mu$ to be the same order of the typical momentum flow of the process or some function of the particles momenta to eliminate

\footnotetext{
*mayangluon@pitt.edu

†wuxg@cqu.edu.cn
}

Published by the American Physical Society under the terms of the Creative Commons Attribution 4.0 International license. Further distribution of this work must maintain attribution to the author(s) and the published article's title, journal citation, and DOI. Funded by SCOAP ${ }^{3}$. large logs and achieve a better pQCD convergence, and takes an arbitrary range to estimate the uncertainties in the fixed-order QCD prediction. However, there is no guarantee that the actual fixed-order $\mathrm{PQCD}$ prediction lies within the assumed range.

When $n \rightarrow \infty$, the infinite series $\varrho_{n \rightarrow \infty}$ corresponds to the exact value of the observable and is independent to the choices of renormalization scheme and scale. This is the standard renormalization group (RG) invariance. However, this RG invariance is caused by compensation of scheme or scale dependence for all orders; thus, at any finite order, the renormalization scheme and scale dependence from the strong coupling constant and the perturbative coefficients do not exactly cancel, leading to the well-known ambiguities. The fixed-order prediction obtained by using the above mentioned guessed scale depends heavily on the renormalization scheme which is itself arbitrary. Thus, a primary problem for $\mathrm{pQCD}$ is how to set the renormalization scale so as to obtain the most accurate fixed-order estimate while satisfying the principles of the renormalization group.

A solution of such ambiguities can be achieved by the exact cancellation of scheme-and-scale dependence at each perturbative order, which can be done by applying the "principle of maximum conformality" (PMC) [1-4]; two comprehensive reviews on PMC together with its 
applications can be found in Refs. [5,6]. The PMC is theoretically sound, but its procedures are somewhat complex and depend heavily on how well we know the nonconformal terms of the $\mathrm{pQCD}$ series.

It is interesting to know whether there are other approaches which can achieve the same goal. In the paper, we shall concentrate our attention on another solution suggested in the literature, i.e. the "principle of minimum sensitivity" (PMS) [7], which sets the optimal renormalization scale and renormalization scheme by directly requiring the slope of the $\mathrm{pQCD}$ approximant over the scheme and scale changes vanish. The PMS, which does not satisfy the RG properties of symmetry, reflexivity, and transitivity [8], gives an incorrect prediction in the lowenergy region [9]. It could be a practical way to achieve a reliable $\mathrm{pQCD}$ prediction when enough higher-order terms are included.

Before we go any further, we first give some explanations on the RG equation, or the so-called $\beta^{\mathcal{R}}$-function, which is important for all scale-setting approaches. Generally, the scale running behavior of $\alpha_{s}^{\mathcal{R}}(\mu)$ is controlled by the following $\beta^{\mathcal{R}}$-function:

$$
\beta^{\mathcal{R}}=\mu^{2} \frac{\partial}{\partial \mu^{2}}\left(\frac{\alpha_{s}^{\mathcal{R}}(\mu)}{4 \pi}\right)=-\sum_{i=0}^{\infty} \beta_{i}^{\mathcal{R}}\left(\frac{\alpha_{s}^{\mathcal{R}}(\mu)}{4 \pi}\right)^{i+2}
$$

Using the decoupling theorem, only the first two loop terms $\beta_{0}$ and $\beta_{1}$ are scheme independent [10-15], while the highorder $\left\{\beta_{m \geq 2}^{\mathcal{R}}\right\}$-terms are scheme dependent, which are now calculated up to the five-loop level within the conventional $\overline{\mathrm{MS}}$ scheme [16-21].

Following the idea of effective coupling approach [22], any physical observable can be equivalently expressed by an effective coupling which satisfies the similar RG equation. Thus the $\left\{\beta_{m \geq 2}^{\mathcal{R}}\right\}$-terms in the $\mathrm{pQCD}$ approximant of a physical observable can be inversely adopted to get the correct running behavior of the coupling constant. For the PMC scale-setting approach, if one can tick out which $\left\{\beta_{i}^{\mathcal{R}}\right\}$-terms pertain to which perturbative order, one can achieve the correct running behavior by using the RG equation and set the correct scale for the strong coupling at this particular order. However, if it is hard to do such $\left\{\beta_{m \geq 2}^{\mathcal{R}}\right\}$-terms distribution, it is helpful to find a proper approach to deal with them as a whole.

Different renormalization schemes lead to different $\left\{\beta_{m \geq 2}^{\mathcal{R}}\right\}$-terms, thus those $\left\{\beta_{m \geq 2}^{\mathcal{R}}\right\}$-terms can be inversely adopted to characterize a renormalization scheme. For the purpose, Refs. [7,23] extended the RG equation to the extended RG equations to incorporate both the scalerunning and scheme-running behaviors of the coupling, especially via this way the strong coupling at different scales and schemes can be reliably related via a continuous way, since along the evolution trajectory described by the extended RG equations, no dissimilar scales or schemes are involved.

\section{TRANSFORMATION OF pQCD PREDICTION FROM ONE SCHEME TO ANOTHER SCHEME}

As a practical treatment, one suggests that we can get optimal scheme and scale by requiring the $\mathrm{PQCD}$ approximate at any fixed order be independent of the "unphysical" theoretical conventions. This is also the key idea of PMS, which indicates that all the scheme-and-scale dependence of a fixed-order prediction are treated as the negligible high-order effect,

$$
\partial \varrho_{n} / \partial(\mathrm{RS})=\mathcal{O}\left(a_{s}^{p+n+1}\right) \sim 0,
$$

where RS stands for the scheme or scale parameters. Equivalently, it requires the fixed-order approximant $\varrho_{n}$ to satisfy the local RG invariance $[6,24]$

$$
\begin{gathered}
\frac{\partial \varrho_{n}}{\partial \tau}=0, \\
\frac{\partial \varrho_{n}}{\partial \beta_{m}^{\mathcal{R}}}=0, \quad(2 \leq m \leq n),
\end{gathered}
$$

where $\tau=\ln \left(\mu^{2} / \tilde{\Lambda}_{\mathrm{QCD}}^{2}\right)$ with the alternated asymptotic QCD scale $\tilde{\Lambda}_{\mathrm{QCD}}=\left(\beta_{1} / \beta_{0}^{2}\right)^{-\beta_{1} / 2 \beta_{0}^{2}} \Lambda_{\mathrm{QCD}}^{\mathcal{R}}$.

The integration constants of those differential equations, i.e. Eqs. (4) and (5), are scheme-and-scale independent RG invariants. For example, up to the $\mathrm{N}^{3} \mathrm{LO}$ level, there are three RG invariants,

$$
\begin{gathered}
\rho_{1}=\frac{1}{4} p \beta_{0} \tau-\mathcal{C}_{1}^{\mathcal{R}}, \\
\rho_{2}=\mathcal{C}_{2}^{\mathcal{R}}-\frac{(1+p)\left(\mathcal{C}_{1}^{\prime \mathcal{R}}\right)^{2}}{2 p}-\frac{\beta_{1} \mathcal{C}_{1}^{\prime \mathcal{R}}}{4 \beta_{0}}+\frac{p \beta_{2}^{\mathcal{R}}}{16 \beta_{0}}, \\
\rho_{3}=2 \mathcal{C}_{3}^{\prime \mathcal{R}}+\frac{\left(\mathcal{C}_{1}^{\prime \mathcal{R}}\right)^{2} \beta_{1}}{4 p \beta_{0}}-\frac{\mathcal{C}_{1}^{\prime \mathcal{R}} \beta_{2}^{\mathcal{R}}}{8 \beta_{0}}+\frac{p \beta_{3}^{\mathcal{R}}}{64 \beta_{0}} \\
+\frac{2(1+p)(2+p)\left(\mathcal{C}_{1}^{\prime \mathcal{R}}\right)^{3}}{3 p^{2}}-\frac{2(2+p) \mathcal{C}_{1}^{\prime \mathcal{R}} \mathcal{C}_{2}^{\prime \mathcal{R}}}{p},
\end{gathered}
$$

where $\mathcal{C}_{i}^{\prime}=\mathcal{C}_{i} / \mathcal{C}_{0}$. In combination with the known $\mathrm{RG}$ invariants, the local RG equations (4) and (5), and the solution of the RG equation (2) up to the same order as the pQCD approximant, we are ready to derive all the wanted optimal parameters. At high orders, it can be done numerically by using the "spiraling" method [25,26].

It is noted that those RG invariants are also helpful for transforming the pQCD approximant $\varrho_{n}$ under the $\mathcal{R}$ scheme to the one under any other scheme (labeled as the $\mathcal{S}$ scheme). More explicitly, this transformation can be achieved by applying the following two transformations simultaneously: 


$$
a_{s}^{\mathcal{R}} \rightarrow a_{s}^{\mathcal{S}} \quad \text { and } \quad \mathcal{C}_{i}^{\mathcal{R}} \rightarrow \mathcal{C}_{i}^{\mathcal{S}}
$$

The coupling constant $a_{s}^{\mathcal{S}}$ can be derived from $a_{s}^{\mathcal{R}}$ by using the extended RG equations, and the scheme-dependent $\beta_{i \geq 2}^{\mathcal{S}}$-terms which determine $a_{s}^{\mathcal{S}}$ scale running behavior can be achieved by using the relation,

$$
\beta^{\mathcal{S}}\left(a_{s}^{\mathcal{S}}\right)=\left(\partial a_{s}^{\mathcal{S}} / \partial a_{s}^{\mathcal{R}}\right) \beta^{\mathcal{R}}\left(a_{s}^{\mathcal{R}}\right) .
$$

The coefficients $\mathcal{C}_{i}^{\mathcal{S}}$ can be obtained from the coefficients $\mathcal{C}_{i}^{\mathcal{R}}$ by using the RG invariants $\rho_{i}$, e.g. up to the $\mathrm{N}^{3} \mathrm{LO}$ level, we have

$$
\begin{gathered}
\mathcal{C}_{1}^{\mathcal{S}}=\mathcal{C}_{1}^{\mathcal{R}}, \\
\mathcal{C}_{2}^{\mathcal{S}}=\mathcal{C}_{2}^{\mathcal{R}}+\frac{p}{16 \beta_{0}}\left(\beta_{2}^{\mathcal{R}}-\beta_{2}^{\mathcal{S}}\right), \\
\mathcal{C}_{3}^{\mathcal{S}}=\mathcal{C}_{3}^{\mathcal{R}}+\frac{p+1}{16 \beta_{0}} \mathcal{C}_{1}^{\mathcal{R}}\left(\beta_{2}^{\mathcal{R}}-\beta_{2}^{\mathcal{S}}\right)+\frac{p}{128}\left(\beta_{3}^{\mathcal{R}}-\beta_{3}^{\mathcal{S}}\right) .
\end{gathered}
$$

So far we have explained how to transform the pQCD predictions from one scheme to another scheme and have completed the description of the PMS calculation technology. In the following, we shall take two four-loop examples to show the scheme dependence of a pQCD approximant under conventional and PMS scale-setting approaches, respectively.

\section{COMPARISONS OF THE pQCD PREDICTIONS UNDER DIFFERENT SCHEMES}

To do the numerical calculation, the initial scheme is taken as the usual $\overline{\mathrm{MS}}$ scheme [27], and the QCD parameter $\Lambda_{\mathrm{QCD}}$ is fixed by $\alpha_{s}\left(M_{Z}\right)=0.1181$ [28].

Example I: $e^{+} e^{-} \rightarrow$ hadrons. - The annihilation of an electron and positron into hadrons provides one of the most precise platforms for testing the running behavior of the strong coupling constant. Its characteristic parameter is the $R$-ratio, whose definition is

$$
\begin{aligned}
R_{e^{+} e^{-}}(Q) & =\frac{\sigma\left(e^{+} e^{-} \rightarrow \text { hadrons }\right)}{\sigma\left(e^{+} e^{-} \rightarrow \mu^{+} \mu^{-}\right)} \\
& =3 \sum_{q} e_{q}^{2}[1+R(Q)],
\end{aligned}
$$

where $Q$ stands for the $e^{+} e^{-}$collision energy at which the $R$-ratio is measured. The $\mathrm{pQCD}$ approximant of $R(Q)$ up to the $\mathrm{N}^{n} \mathrm{LO}$ level under the $\overline{\mathrm{MS}}$ scheme reads

$$
R_{n}\left(Q, \mu_{0}\right)=\sum_{i=0}^{n} \mathcal{C}_{i}^{\overline{\mathrm{MS}}}\left(Q, \mu_{0}\right)\left(a_{s}^{\overline{\mathrm{MS}}}\right)^{i+1}\left(\mu_{0}\right)
$$

where $\mu_{0}$ stands for an arbitrary initial renormalization scale. If setting $\mu_{0}=Q$, the coefficients $\mathcal{C}_{i}^{\overline{\mathrm{MS}}}(Q, Q)$ up to fourth order can be read from Ref. [29]. For any other choice of $\mu_{0}$, we will use RG equation to get the coefficients from $\mathcal{C}_{i}^{\overline{\mathrm{MS}}}(Q, Q)$.

As a reference, we present the recalculated conventional $\mu_{0}$-dependence of $R_{n}\left(Q, \mu_{0}\right)$ under the $\overline{\mathrm{MS}}$ scheme in Fig. 1, where $Q=31.6 \mathrm{GeV}$. A combination of data from $e^{+} e^{-}$colliders gives $R(31.6 \mathrm{GeV})=0.0527 \pm 0.0050$ [30]. Such $\mu_{0}$-dependence as shown in Fig. 1 is rightly the renormalization scale dependence for conventional scale setting, since $\mu \equiv \mu_{0}$ for conventional scale setting. Conventionally, people would like to choose $\mu_{0}=Q$ and vary it in the range $[Q / 2,2 Q]$ to estimate the uncertainty due to scale ambiguity. Figure 1 shows that under conventional scale setting, the low-order results, e.g. $R_{0}$ and $R_{1}$, depend strongly on the renormalization scale, which becomes weaker and weaker when more and more highorder terms have been included. This agrees with the conventional wisdom on the perturbation theory that one would get a desirable renormalization scale invariant result by finishing enough high-order calculations.

Next, we investigate the renormalization scheme dependence of the $\mathrm{pQCD}$ predictions at $\mathrm{N}^{2} \mathrm{LO}$ and beyond. We will also show how the PMS prediction changes with a different choice of initial schemes as a comparison. Since the PMS prediction is $\mu_{0}$-independent [24], it is safe to fix $\mu_{0}=Q$ in the following discussions.

At the $\mathrm{N}^{2} \mathrm{LO}$ level and higher, the scheme dependence of the $\mathrm{pQCD}$ prediction could be equivalently represented by a group of scheme-dependent $\left\{\beta_{m \geq 2}\right\}$-terms, since the $\left\{\beta_{m \geq 2}\right\}$-terms are specific for a specific scheme. At the $\mathrm{N}^{n} \mathrm{LO}$ level, the number of $\left\{\beta_{m \geq 2}\right\}$-terms is $(n-1)$, which characterize the scheme independence of the PMS prediction via the local version of RG equations (5). It is worthy to point out that, unlike the renormalization scale which should be varied in a region that is not too far away from a character value $Q$ of a given process, there is no such

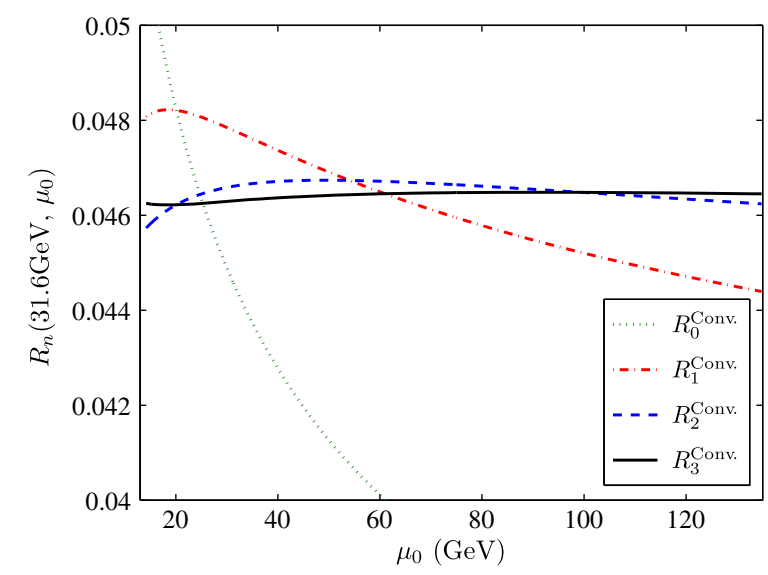

FIG. 1. The pQCD approximant $R_{n}\left(Q=31.6 \mathrm{GeV}, \mu_{0}\right)$ up to four-loop level versus the initial scale $\mu_{0}$. The dotted, the dashdotted, the dashed and the solid lines are for $R_{0}, R_{1}, R_{2}$ and $R_{3}$, respectively. 
constraint for $\left\{\beta_{m \geq 2}\right\}$-terms. In principle one could choose any value for $\left\{\beta_{m \geq 2}\right\}$-terms and define a $\mathcal{R}$ scheme for a given fixed order pQCD calculation, as long as $\beta^{\mathcal{R}}\left(\alpha_{s}(Q)\right)<0$, i.e. the asymptotic freedom is satisfied. Following the idea,

(1) We use $R_{n, m}\left(Q, \beta_{m}\right)$ to replace $R_{n}$ to show explicitly how the scheme-dependent $\beta_{m}$-term affects the $\mathrm{N}^{n} \mathrm{LO}$ prediction $R_{n}$.

(2) When discussing the scheme error from the $\beta_{m}$-term, the other involved $\left\{\beta_{j \neq m}\right\}$-terms shall be fixed to be their $\overline{\mathrm{MS}}$-scheme values.

(3) We adopt a broad range for the allowable $\left\{\beta_{m}\right\}$ terms to numerically discuss the scheme dependence of a $\mathrm{N}^{n} \mathrm{LO}$ prediction, e.g. $\beta_{m} \in\left[-50 \beta_{m}^{\overline{\mathrm{MS}}},+50 \beta_{m}^{\overline{\mathrm{MS}}}\right]$ for $m \in[2, n]$.

The dependence of the ratio $R_{n, m}\left(Q, \beta_{m}\right)$ on $\beta_{m}$ with $m \in[2, n]$ is presented in Fig. 2, where $n=[2,3]$ and $Q=31.6 \mathrm{GeV}$. Here $n=2$ and $n=3$ stand for the $\mathrm{N}^{2} \mathrm{LO}$ and $\mathrm{N}^{3} \mathrm{LO}$ pQCD predictions, respectively. As a comparison, both the results for conventional scale setting and PMS are presented. Under conventional scale setting,

(1) Figure 2 shows the scheme dependence of the pQCD calculation follows the perturbative nature, which drops down when more perturbative terms are included.

(2) Comparing with Fig. 1, Fig. 2 shows the magnitude of the scheme dependence could be much larger than that of the scale dependence:

(a) At the $\mathrm{N}^{2} \mathrm{LO}$ level, the scale dependence is $\sim 2 \%$ for $\mu_{0} \in[Q / 2,2 Q]$, while the scheme dependence is $\sim 4 \%$ for $\beta_{2} \in\left[-10 \beta_{2}^{\overline{\mathrm{MS}}},+10 \beta_{2}^{\overline{\mathrm{MS}}}\right]$ and $\sim 19 \%$ for $\beta_{2} \in\left[-50 \beta_{2}^{\overline{\mathrm{MS}}},+50 \beta_{2}^{\overline{\mathrm{MS}}}\right]$. Figure 2 shows that $R_{2,2}\left(Q, \beta_{2}\right)$ shall first increase and then decrease with the increment of $\beta_{2}$.

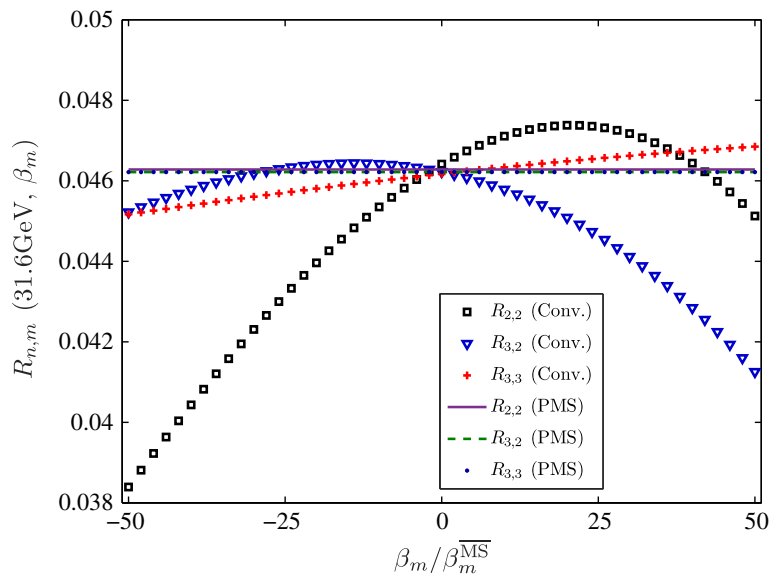

FIG. 2. The pQCD predictions for $R_{n, m}\left(Q, \beta_{m}\right)$ versus the $\beta_{m \geq 2}$-term at the $\mathrm{N}^{2} \mathrm{LO}(n=2)$ and $\mathrm{N}^{3} \mathrm{LO}(n=3)$ levels respectively. $Q=31.6 \mathrm{GeV}$. As a comparison, both the results for conventional scale setting and PMS are presented. (b) At the $\mathrm{N}^{3} \mathrm{LO}$ level, the scale dependence reduces to be $\sim 0.2 \%$, while the scheme dependence is still about $7 \%$ in which an extra $\sim 4 \%$ error comes from the $\beta_{3}$-term within the region of $\left[-50 \beta_{3}^{\overline{\mathrm{MS}}},+50 \beta_{3}^{\overline{\mathrm{MS}}}\right]$. Figure 2 shows $R_{3,2}\left(Q, \beta_{2}\right)$ shall first increase and then decrease with the increment of $\beta_{2}$, and $R_{3,3}\left(Q, \beta_{3}\right)$ shall monotonously increase with the increment of $\beta_{3}$.

The PMS determines the optimal scheme and scale by requiring the slope of the $\mathrm{pQCD}$ prediction to vanish. Figure 2 shows the PMS prediction is stable over the scheme changes. That is, the flat lines for $\left.R_{2,2}\left(Q, \beta_{2}\right)\right|_{\mathrm{PMS}}$, $\left.R_{3,2}\left(Q, \beta_{2}\right)\right|_{\mathrm{PMS}}$ and $\left.R_{3,3}\left(Q, \beta_{3}\right)\right|_{\mathrm{PMS}}$ indicate that the PMS predictions are scheme independent at each order.

Figure 3 shows the combined $\left\{\beta_{2}, \beta_{3}\right\}$-dependence for the $\mathrm{N}^{3} \mathrm{LO}$ prediction $R_{3}$, in which the scheme-dependent $\beta_{2}$ and $\beta_{3}$ terms change simultaneously within the regions of $\left[-50 \beta_{2}^{\overline{\mathrm{MS}}},+50 \beta_{2}^{\overline{\mathrm{MS}}}\right]$ and $\left[-50 \beta_{3}^{\overline{\mathrm{MS}}},+50 \beta_{3}^{\overline{\mathrm{MS}}}\right]$. The flat plane confirms the scheme independence of the PMS prediction over the changes of $\left\{\beta_{2}, \beta_{3}\right\}$, thus by using the scheme equations (5), one cannot only achieve the most stable pQCD prediction around the optimal point (determined by the optimal scheme and the optimal scale) but also achieve the scheme-independent prediction over the choices of $\left\{\beta_{m}\right\}$-terms, or equivalently over different choices of the initial renormalization scheme.

To be more specific, we present the numerical results of $R_{n}$ under three usually adopted schemes, i.e. $\overline{\mathrm{MS}}$ scheme, MOM scheme [31] and V scheme [32], in Table I. Typical values for $\beta_{2}$ and $\beta_{3}$ of those renormalization schemes are presented in Table II. Here, the result for the MOM scheme is obtained by using the Landau gauge and following the method of Ref. [33]; the result for the $\mathrm{V}$ scheme is consistent with Ref. [34]. For $\mu_{0}=Q=31.6 \mathrm{GeV}$, which corresponds to $n_{f}=5$, we have

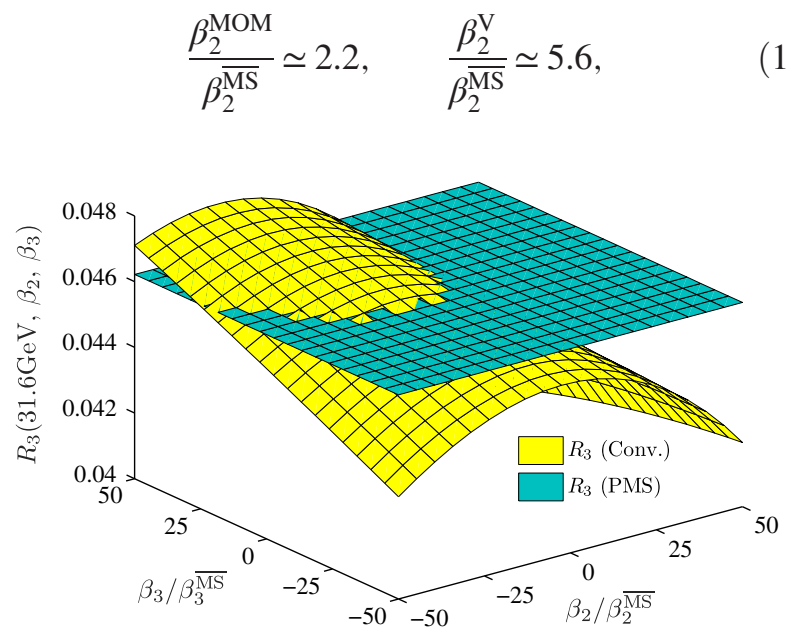

FIG. 3. Comparison of the combined $\left\{\beta_{2}, \beta_{3}\right\}$-dependence for the $\mathrm{N}^{3} \mathrm{LO}$ prediction $R_{3}$ under the conventional (Conv.) scale setting and PMS, respectively. $Q=31.6 \mathrm{GeV}$. 
TABLE I. Numerical results for $R_{n}$ and $\kappa_{n}$ with various QCD loop corrections under the conventional scale setting. $\mu_{0}=Q$ and $Q=31.6 \mathrm{GeV}$. Three renormalization schemes, $\overline{\mathrm{MS}}$, MOM and $\mathrm{V}$, are adopted for a comparison.

\begin{tabular}{lcccccc}
\hline \hline & $R_{1}$ & $R_{2}$ & $R_{3}$ & $\kappa_{1}$ & $\kappa_{2}$ & $\kappa_{3}$ \\
\hline$\overline{M S}$ (Conv.) & 0.04765 & 0.04650 & 0.04619 & $\ldots$ & $\ldots$ & $\ldots$ \\
MOM (Conv.) & 0.04810 & 0.04604 & 0.04608 & $0.9 \%$ & $1.0 \%$ & $0.2 \%$ \\
V (Conv.) & 0.04801 & 0.04653 & 0.04587 & $0.8 \%$ & $0.1 \%$ & $0.7 \%$ \\
\hline \hline
\end{tabular}

TABLE II. Numerical values for $\beta_{2}$ and $\beta_{3}$ under the three renormalization schemes, $\overline{\mathrm{MS}}, \mathrm{MOM}$ and $\mathrm{V}$, respectively.

\begin{tabular}{lllc}
\hline \hline & $n_{f}=3$ & $n_{f}=4$ & $n_{f}=5$ \\
\hline$\beta_{2}^{\overline{\mathrm{MS}}}$ & 643.833 & 406.352 & 180.907 \\
$\beta_{2}^{\mathrm{MOM}}$ & 1338.77 & 849.069 & 398.132 \\
$\beta_{2}^{\mathrm{V}}$ & 2174.01 & 1574.11 & 1015.95 \\
$\beta_{3}^{\overline{\mathrm{MS}}}$ & 12090.4 & 8035.19 & 4826.16 \\
$\beta_{3}^{\mathrm{MOM}}$ & 41157.4 & 27094.6 & 15622.9 \\
$\beta_{3}^{\mathrm{V}}$ & 10537.9 & 2355.74 & -4529.47 \\
\hline \hline
\end{tabular}

$$
\frac{\beta_{3}^{\mathrm{MOM}}}{\beta_{3}^{\overline{\mathrm{MS}}}} \simeq 3.2, \quad \frac{\beta_{3}^{\mathrm{V}}}{\beta_{3}^{\overline{\mathrm{MS}}}} \simeq-0.9
$$

In Table I, to show the scheme dependence, we have defined the ratio $\kappa_{n}^{\mathcal{R}}$ for a specific scheme $\mathcal{R}$ :

$$
\kappa_{n}^{\mathcal{R}}=\left|\frac{R_{n}^{\mathcal{R}}-R_{n}^{\overline{\mathrm{MS}}}}{R_{n}^{\overline{\mathrm{MS}}}}\right|, \quad(n \in[1,3]) .
$$

Under conventional scale setting, by setting $\mu_{0}=Q$, we observe that $\kappa_{1}^{\mathrm{MOM}, \mathrm{V}} \sim 1 \%$ for $Q=31.6 \mathrm{GeV}$, which generally drops down as more loop terms come into contribution. However, such a shrink tendency heavily depends on the cancellations among different perturbative orders which could be accidental. For example, $\kappa_{3}^{\mathrm{V}} \sim \kappa_{1}^{\mathrm{V}}$ and $\kappa_{3}^{\mathrm{V}} \gg \kappa_{2}^{\mathrm{V}}$. This anomaly can be qualitatively explained by the ascending trends of $R_{n, m}\left(Q, \beta_{m}\right)$ versus the $\left\{\beta_{m \geq 2}\right\}$-terms as shown by Fig. $2, R_{3,2}\left(Q, \beta_{2}\right)$ first increases and then decreases with the increment of $\beta_{2}$, and $R_{3,3}\left(Q, \beta_{3}\right)$ monotonously increases with the increment of $\beta_{3}$. Thus the fact of $\beta_{2}^{\mathrm{V}}>\beta_{2}^{\overline{\mathrm{MS}}}$ and $\beta_{3}^{\mathrm{V}}<\beta_{3}^{\overline{\mathrm{MS}}}$ indicates there is no cancellation between $\beta_{2}^{\mathrm{V}}$-terms and $\beta_{3}^{\mathrm{V}}$-terms at the $\mathrm{N}^{3} \mathrm{LO}$ level, leading to a larger difference between $R_{3}^{\mathrm{V}}=0.04587$ and $R_{3}^{\overline{\mathrm{MS}}}=0.04619$.

On the other hand, being consistent with previous observations, after applying PMS scale setting, the scheme dependence is eliminated and all three schemes lead to the same predictions, ${ }^{1}$

$R_{1}=0.04868, \quad R_{2}=0.04628, \quad R_{3}=0.04622$.

Example II: $\tau$ decays into hadrons.-The $\tau$ decays into hadrons is another important platform to test the pQCD theory, whose characteristic parameter is the following ratio:

$$
\begin{aligned}
R_{\tau}\left(M_{\tau}\right) & =\frac{\Gamma\left(\tau \rightarrow \nu_{\tau}+\text { hadrons }\right)}{\Gamma\left(\tau \rightarrow \nu_{\tau}+e^{-} \bar{\nu}_{e}\right)} \\
& =3\left(\left|V_{u d}\right|^{2}+\left|V_{u s}\right|^{2}\right)\left[1+r^{\tau}\left(M_{\tau}\right)\right],
\end{aligned}
$$

where the $\tau$-lepton mass $M_{\tau}=1.777 \mathrm{GeV}$ [28] and the Cabbibo-Kobayashi-Maskawa matrix elements $V_{u d}$ and $V_{u s}$ satisfy the relation, $3\left(\left|V_{u d}\right|^{2}+\left|V_{u s}\right|^{2}\right) \approx 3$. The pQCD approximant of $r^{\tau}\left(M_{\tau}\right)$ up to the $\mathrm{N}^{n} \mathrm{LO}$ level under the $\overline{\mathrm{MS}}$ scheme reads

$$
r_{n}^{\tau}\left(M_{\tau}, \mu_{0}\right)=\sum_{i=0}^{n} \mathcal{C}_{i}^{\overline{\mathrm{MS}}}\left(M_{\tau}, \mu_{0}\right)\left(a_{s}^{\overline{\mathrm{MS}}}\right)^{i+1}\left(\mu_{0}\right) .
$$

The perturbative coefficients up to fourth order at any scale $\mu_{0}$ can be derived from the ones in Ref. [35].

Using the above formulas, we calculate the initial scale dependence of $r_{n}^{\tau}\left(M_{\tau}, \mu_{0}\right)$ and present it in Fig. 4, which shows a much larger scale dependence than that of $R_{n}\left(Q, \mu_{0}\right)$. The reason for this larger scale dependence even up to the four-loop level is due to the poorer pQCD convergence of the conventional pQCD series, which is caused by the divergent renormalon terms in combination of a somewhat larger $\alpha_{s}$ value at the lower scale around $M_{\tau}$ that is not far from $\Lambda_{\mathrm{QCD}}$. We observe that at the four-loop level, under conventional scale setting, the scale dependence is still about $25 \%$ for $\mu_{0} \in\left[M_{\tau} / 2,2 M_{\tau}\right]$. Thus an even higher order calculation is necessary to further suppress the conventional scale uncertainty. After applying PMS scale setting, the PMS prediction on $R_{n}\left(Q, \mu_{0}\right)$ is independent to the choice of $\mu_{0}$, and for convenience, we set $\mu_{0}=M_{\tau}$ to do the following discussion.

Similarly, we use $r_{n, m}^{\tau}\left(M_{\tau}, \beta_{m}\right)$ instead of $r_{n}^{\tau}$ to discuss the scheme dependence. The magnitude of the conventional scheme dependence is large, so we adopt a smaller region, $\beta_{m} \in\left[-5 \beta_{m}^{\overline{\mathrm{MS}}},+5 \beta_{m}^{\overline{\mathrm{MS}}}\right]$ for the discussion. The results for the $\beta_{m}$ dependence of $r_{n, m}^{\tau}\left(M_{\tau}, \beta_{m}\right)$ before and after PMS scale setting are presented in Figs. 5 and 6, where $n \in[2,3]$.

\footnotetext{
${ }^{1}$ The four-loop pQCD prediction before and after applying PMS scale setting gives $R(31.6 \mathrm{GeV}) \sim 0.046$, which is smaller than the measured value $[0.0477,0.0557]$ [30]. A precise determination of $\Lambda_{\mathrm{QCD}}$ by using the $e^{+} e^{-}$data alone shall be helpful for explanation of such a difference, which is in preparation.
} 


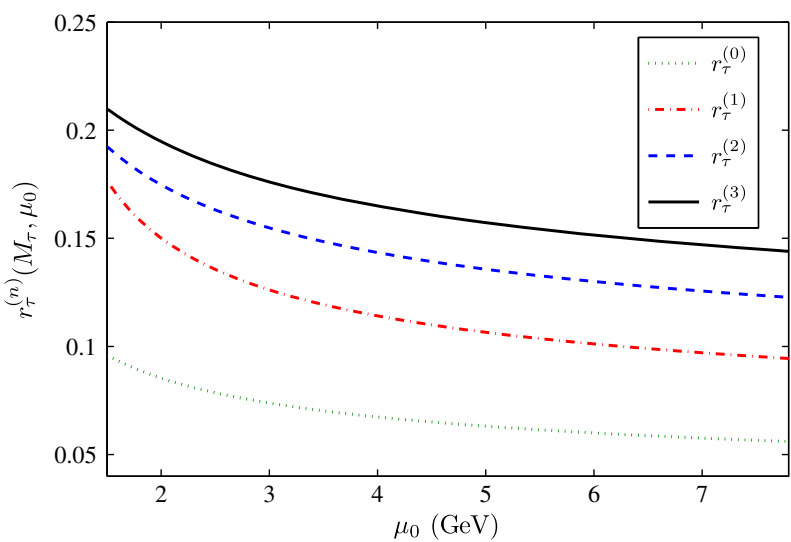

FIG. 4. The pQCD approximant $r_{n}^{\tau}\left(M_{\tau}, \mu_{0}\right)$ up to four-loop level versus the initial scale $\mu_{0}$. The dotted, the dash-dotted, the dashed, and the solid lines are for $r_{0}^{\tau}, r_{1}^{\tau}, r_{2}^{\tau}$ and $r_{3}^{\tau}$, respectively.

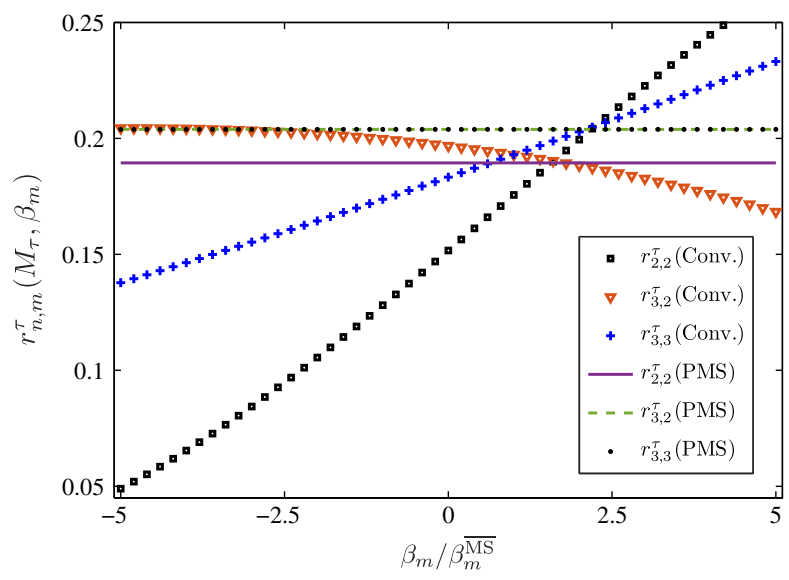

FIG. 5. The pQCD predictions for $r_{n}^{\tau}\left(M_{\tau}, \beta_{m}\right)$ versus the $\beta_{m \geq 2^{-}}$ term at the $\mathrm{N}^{2} \mathrm{LO}(n=2)$ and $\mathrm{N}^{3} \mathrm{LO}(n=3)$ levels respectively. As a comparison, both the results for conventional scale setting and PMS are presented.

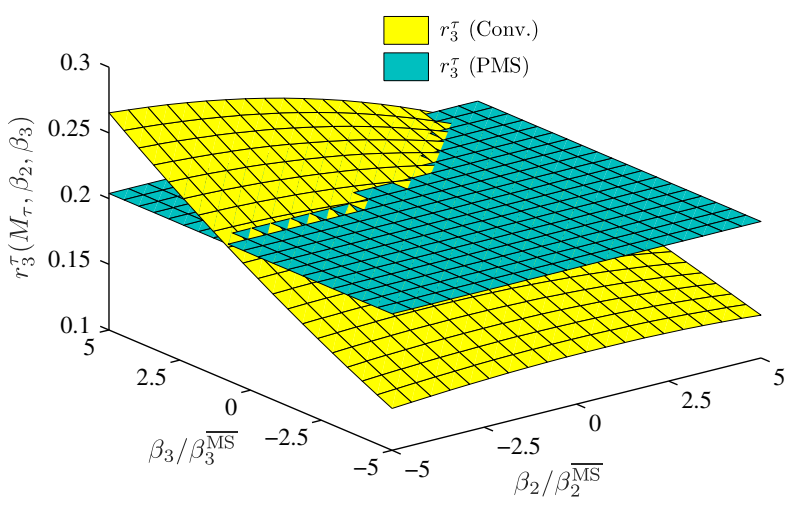

FIG. 6. Comparison of the combined $\left\{\beta_{2}, \beta_{3}\right\}$-dependence for the $\mathrm{N}^{3} \mathrm{LO}$ prediction $r_{3}^{\tau}$ under the conventional (Conv.) scale setting and PMS, respectively.
TABLE III. Numerical results for $r_{n}^{\tau}$ and $\kappa_{n}^{\tau}$ with various QCD loop corrections under the conventional scale setting. $\mu_{0}=M_{\tau}$. Three renormalization schemes, $\overline{\mathrm{MS}}, \mathrm{MOM}$ and $\mathrm{V}$, are adopted for a comparison.

\begin{tabular}{lcccccc}
\hline \hline & $r_{1}^{\tau}$ & $r_{2}^{\tau}$ & $r_{3}^{\tau}$ & $\kappa_{1}^{\tau}$ & $\kappa_{2}^{\tau}$ & $\kappa_{3}^{\tau}$ \\
\hline$\overline{\mathrm{MS}}$ (Conv.) & 0.1544 & 0.1755 & 0.1930 & $\ldots$ & $\ldots$ & $\ldots$ \\
MOM (Conv.) & 0.2389 & 0.2581 & 0.1423 & $55 \%$ & $47 \%$ & $26 \%$ \\
$\mathrm{~V}$ (Conv.) & 0.1975 & 0.2773 & 0.1719 & $28 \%$ & $58 \%$ & $11 \%$ \\
\hline \hline
\end{tabular}

To compare with Fig. 2, Fig. 5 shows that $r_{n, m}^{\tau}\left(M_{\tau}, \beta_{m}\right)$ has a much heavier $\beta$-dependence than that of $R_{n, m}\left(Q, \beta_{m}\right)$, which increases (decreases) monotonously with the increment of $\beta_{2}\left(\beta_{3}\right)$. At the $\mathrm{N}^{3} \mathrm{LO}$ level, the conventional scheme uncertainty is about $30 \%$ for $\beta_{2} \in\left[-5 \beta_{2}^{\overline{\mathrm{MS}}},+5 \beta_{2}^{\overline{\mathrm{MS}}}\right]$ and $\beta_{3} \in\left[-5 \beta_{3}^{\overline{\mathrm{MS}}},+5 \beta_{3}^{\overline{\mathrm{MS}}}\right]$. The flat lines in Fig. 5 and the flat plane in Fig. 6 show the PMS predictions are stable over the scheme changes.

Typical results of $r_{n}^{\tau}$ and $\kappa_{n}^{\tau}$ under the $\overline{\mathrm{MS}}$ scheme, the MOM scheme and the V scheme are presented in Table III. On the other hand, being consistent with the previous observations, after applying PMS scale setting, the scheme dependence is eliminated and all three schemes lead to the same predictions:

$r_{1}^{\tau}=0.3238, \quad r_{2}^{\tau}=0.1894, \quad r_{3}^{\tau}=0.2039$.

\section{SUMMARY}

We have investigated the renormalization scheme dependence of high-order $\mathrm{pQCD}$ predictions via studying the sensitivity of the pQCD calculations on the renormalization scheme parameters $\left\{\beta_{m \geq 2}^{\mathcal{R}}\right\}$. For a fixed-order prediction, by simply using a guessed scale as done by conventional scale setting, there are renormalization scheme and scale ambiguities. Those ambiguities could be softened by finishing more and more loop terms.

We observe that, different to the scale dependence, because new scheme-dependent $\beta$-terms emerge at higher orders which introduce new scheme dependence into the prediction, the scheme dependence tends to be dropped down much slower. For example, the scale dependence of the $\mathrm{N}^{2} \mathrm{LO}$ prediction $R_{2}$ is around $2 \%$ for $\mu \in[Q / 2,2 Q]$ with $Q=31.6 \mathrm{GeV}$, while the scheme dependence is still $\sim 19 \%$ for $\beta_{2} \in\left[-50 \beta_{2}^{\overline{\mathrm{MS}}},+50 \beta_{2}^{\overline{\mathrm{MS}}}\right]$. Moving to the $\mathrm{N}^{3} \mathrm{LO}$ level, the scale dependence of $R_{3}$ reduces to be less than $0.2 \%$ for $\mu \in[Q / 2,2 Q]$ with $Q=31.6 \mathrm{GeV}$ and the scheme dependence is $\sim 7 \%$ for $\beta_{2} \in\left[-50 \beta_{2}^{\overline{\mathrm{MS}}},+50 \beta_{2}^{\overline{\mathrm{MS}}}\right]$ and $\beta_{3} \in\left[-50 \beta_{3}^{\overline{\mathrm{MS}}},+50 \beta_{3}^{\overline{\mathrm{MS}}}\right]$; meanwhile, the scale dependence of $r_{3}^{\tau}$ is $\sim 25 \%$ for $\mu \in\left[M_{\tau} / 2,2 M_{\tau}\right]$ and the scheme dependence is about $30 \%$ for $\beta_{2} \in\left[-5 \beta_{2}^{\overline{\mathrm{MS}}},+5 \beta_{2}^{\overline{\mathrm{MS}}}\right]$ and $\beta_{3} \in\left[-5 \beta_{3}^{\overline{\mathrm{MS}}},+5 \beta_{3}^{\overline{\mathrm{MS}}}\right]$. 
We have adopted three schemes, the $\overline{\mathrm{MS}}$ scheme, the MOM scheme and the V scheme, to show how the scheme dependence changes when more loop terms are included. Tables I and III show $\kappa_{3}^{\mathrm{V}} \sim \kappa_{1}^{\mathrm{V}}, \kappa_{3}^{\mathrm{V}} \gg \kappa_{2}^{\mathrm{V}}$ for $R$ and $\kappa_{2}^{\mathrm{MOM}} \sim \kappa_{1}^{\mathrm{MOM}}, \kappa_{2}^{\mathrm{V}} \gg \kappa_{1}^{\mathrm{V}}$ for $r^{\tau}$, reflecting that those high-order terms cannot effectively suppress the scheme uncertainty as far as they do for the scale uncertainty.

As a summary, it has been found that the elimination of the scheme dependence is as important as the elimination of the scale dependence. It is important to find a proper approach to deal with both issues simultaneously. The PMS treats the fixed-order $\mathrm{pQCD}$ prediction as the exact prediction of the physical observable, which breaks the RG invariance. Figures 2, 3, 5, and 6 show that by applying the PMS, one can achieve scheme-and-scale independent predictions with the help of RG invariants such as those of Eqs. (6)-(8). Even though the PMS cannot offer correct lower-order predictions [24], it shall provide a reliable prediction when enough higher-order terms are included. Thus, in certain cases when the pQCD series has a good convergence, the PMS could be a practical approach to soften the renormalization scheme and scale ambiguities for high-order $\mathrm{pQCD}$ predictions.

\section{ACKNOWLEDGMENTS}

We thank Hua-Yong Han and Xu-Chang Zheng for helpful discussions. This work was supported in part by National Natural Science Foundation of China under Grant No. 11625520. PITT PACC-1708.
[1] S. J. Brodsky and X.G. Wu, Scale setting using the extended renormalization group and the principle of maximum conformality: The QCD coupling constant at four loops, Phys. Rev. D 85, 034038 (2012).

[2] S. J. Brodsky and X. G. Wu, Eliminating the Renormalization Scale Ambiguity for Top-Pair Production Using the Principle of Maximum Conformality, Phys. Rev. Lett. 109, 042002 (2012).

[3] M. Mojaza, S. J. Brodsky, and X. G. Wu, Systematic AllOrders Method to Eliminate Renormalization-Scale and Scheme Ambiguities in Perturbative QCD, Phys. Rev. Lett. 110, 192001 (2013).

[4] S. J. Brodsky, M. Mojaza, and X. G. Wu, Systematic scalesetting to all orders: The principle of maximum conformality and commensurate scale relations, Phys. Rev. D 89, 014027 (2014).

[5] X. G. Wu, S. J. Brodsky, and M. Mojaza, The renormalization scale-setting problem in QCD, Prog. Part. Nucl. Phys. 72, 44 (2013).

[6] X. G. Wu, Y. Ma, S. Q. Wang, H. B. Fu, H. H. Ma, S. J. Brodsky, and M. Mojaza, Renormalization group invariance and optimal QCD renormalization scale setting, Rep. Prog. Phys. 78, 126201 (2015).

[7] P. M. Stevenson, Optimized perturbation theory, Phys. Rev. D 23, 2916 (1981).

[8] S. J. Brodsky and X. G. Wu, Self-consistency requirements of the renormalization group for setting the renormalization scale, Phys. Rev. D 86, 054018 (2012).

[9] G. Kramer and B. Lampe, Optimized perturbation theory applied to jet cross sections in $e^{+} e^{-}$annihilation, Z. Phys. C 39, 101 (1988).

[10] H. D. Politzer, Reliable Perturbative Results for Strong Interactions?, Phys. Rev. Lett. 30, 1346 (1973).

[11] C. G. Callan, Jr., Broken scale invariance in scalar field theory, Phys. Rev. D 2, 1541 (1970).

[12] I. B. Khriplovich, Green's functions in theories with nonAbelian gauge group, Yad. Fiz. 10, 409 (1969) [Sov. J. Nucl. Phys. 10, 235 (1969)].
[13] W. E. Caswell, Asymptotic Behavior of Non-Abelian Gauge Theories to Two Loop Order, Phys. Rev. Lett. 33, 244 (1974).

[14] D. R. T. Jones, Two loop diagrams in Yang-Mills theory, Nucl. Phys. B75, 531 (1974).

[15] E. Egorian and O. V. Tarasov, Two loop renormalization of the QCD in an arbitrary gauge, Teor. Mat. Fiz. 41, 26 (1979) [Theor. Math. Phys. 41, 863 (1979)].

[16] O. V. Tarasov, A. A. Vladimirov, and A. Y. Zharkov, The Gell-Mann-Low function of QCD in the three loop approximation, Phys. Lett. 93B, 429 (1980).

[17] S. A. Larin and J. A. M. Vermaseren, The three loop QCD beta function and anomalous dimensions, Phys. Lett. B 303, 334 (1993).

[18] T. van Ritbergen, J. A. M. Vermaseren, and S. A. Larin, The four loop beta function in quantum chromodynamics, Phys. Lett. B 400, 379 (1997).

[19] K. G. Chetyrkin, Four-loop renormalization of QCD: Full set of renormalization constants and anomalous dimensions, Nucl. Phys. B710, 499 (2005).

[20] M. Czakon, The four-loop QCD beta function and anomalous dimensions, Nucl. Phys. B710, 485 (2005).

[21] P. A. Baikov, K. G. Chetyrkin, and J. H. Kuhn, Five-Loop Running of the QCD Coupling Constant, Phys. Rev. Lett. 118, 082002 (2017).

[22] G. Grunberg, Renormalization scheme independent QCD and QED: The method of effective charges, Phys. Rev. D 29, 2315 (1984).

[23] H. J. Lu and S. J. Brodsky, Relating physical observables in QCD without scale-scheme ambiguity, Phys. Rev. D 48, 3310 (1993).

[24] Y. Ma, X. G. Wu, H. H. Ma, and H. Y. Han, General properties on applying the principle of minimum sensitivity to high-order perturbative QCD predictions, Phys. Rev. D 91, 034006 (2015).

[25] A. C. Mattingly and P. M. Stevenson, QCD Perturbation Theory at Low Energies, Phys. Rev. Lett. 69, 1320 (1992). 
[26] A.C. Mattingly and P. M. Stevenson, Optimization of $\mathrm{R}(\mathrm{e}+\mathrm{e}-)$ and "freezing" of the QCD couplant at low energies, Phys. Rev. D 49, 437 (1994).

[27] W. A. Bardeen, A. J. Buras, D. W. Duke, and T. Muta, Deep inelastic scattering beyond the leading order in asymptotically free gauge theories, Phys. Rev. D 18, 3998 (1978).

[28] C. Patrignani et al. (Particle Data Group), Review of particle physics, Chin. Phys. C 40, 100001 (2016).

[29] P. A. Baikov, K. G. Chetyrkin, J. H. Kuhn, and J. Rittinger, Adler function, sum rules and Crewther relation of order $O\left(\alpha_{s}^{4}\right)$ : The singlet case, Phys. Lett. B 714, 62 (2012).

[30] R. Marshall, A determination of the strong coupling constant $\alpha^{-} s$ from $e^{+} e^{-}$total cross-section data, Z. Phys. C 43, 595 (1989).
[31] W. Celmaster and R. J. Gonsalves, QCD Perturbation Expansions in a Coupling Constant Renormalized by Momentum Space Subtraction, Phys. Rev. Lett. 42, 1435 (1979).

[32] M. Peter, The Static Quark-Anti-Quark Potential in QCD to Three Loops, Phys. Rev. Lett. 78, 602 (1997).

[33] K. G. Chetyrkin and A. Retey, Three loop three linear vertices and four loop similar to MOM beta functions in massless QCD, arXiv:hep-ph/0007088.

[34] A. L. Kataev and V.S. Molokoedov, Fourth-order QCD renormalization group quantities in the $\mathrm{V}$ scheme and the relation of the $\beta$ function to the Gell-Mann-Low function in QED, Phys. Rev. D 92, 054008 (2015).

[35] P. A. Baikov, K. G. Chetyrkin, and J. H. Kuhn, Order $\alpha^{4}(s)$ QCD Corrections to Z and tau Decays, Phys. Rev. Lett. 101, 012002 (2008). 